\title{
Design of Broadband Transition Based on Substrate Integrated Waveguide
}

\author{
Qinghua Han and Mingdeng Shi* \\ College of Information Engineering, Tarim University, China \\ ${ }^{*}$ Corresponding author
}

\begin{abstract}
A broadband transition device based on substrate integrated waveguide structureis proposed in this letter. With the novel guide wave structure, electromagnetic waves could be limited in the rectangular cavity formed by two rows of metal hole and the upper and lower metal border to achieve low losseffect. Combined with the advantages of that multiple impedance transformer, it can broaden the bandwidth of microwave circuits. A broadband transition is designed to cover the $33 \mathrm{GHz}-50 \mathrm{GHz}$ band showing a return loss of $10 \mathrm{~dB}$ and insertion loss of $0.68 \mathrm{~dB}$. The measurement error is within an allowable range and consistents with the simulation results. The proposed transition meets the design requirements for broadband and low-loss.
\end{abstract}

Keywords-SIW; multiple impedance transformer; broadband

\section{INTRODUCTION}

Guided wave structures is the basis of microwave circuit, its transmission characteristics and stable properties has a direct impact on the performance of the microwave circuit.From the beginning the study of metal waveguide structure to the development of the microstrip circuit ${ }^{[1]}$.People summarized the integration problem between circuit and analyzed the future development trend, this paper proposes a waveguide structure based on dielectric substrate-Substrate Integrated Waveguide ${ }^{[2]}$, (SIW).SIW in addition to comprehensive metal waveguide structure and advantages of microstrip circuit, it also has a high quality factor and high integration ability, at the same time can reduce the manufacturing cost. It widely used in military communication, ground radar, and other fields, and yet in many special fields as the preferred parts, it has become one of the important research direction of microwave circuit in recent years. Due to the need of impedance matching ${ }^{[3-4]}$, impedance transformation is necessary, but at higher in RF and microwave band, it is difficult to achieve broadband for larger impedance transformation.Through the reflection and discontinuity theory to design of device, by increasing the impedance converter session can be made on wide band to obtain the ideal matching features.

This article through to the study of substrate integrated waveguide and the theory and structure of the impedance converter. A broadband transition is designed.the transition device has the advantages of wide band, low loss.

\section{BASIC KNOWLEDGE AND DESIGN WAVEGUIDE TRANSITION}

\section{A. Substrate Integrated Waveguide Theory and Design}

The basic structure of the substrate integrated waveguide is in double sided PCB dielectric plate coated copper perpendicular to insert two rows of parallel with periodic metal hole clearance, and the upper and lower sides of the metal surface of PCB board.Because each row metal hole hole spacing is far smaller than the wavelength, therefore Small aperture leakage of energy, equivalent to the rectangular waveguide filled the medium. the most parts of the electromagnetic energy is limited within the space between the metal wall.

To achieve good loss within the wide band, two transitions.wide-band rectangular waveguide converter and SIW with microstripline.wide-band converter in high frequencies, its high order modes of the coaxial connector joint converter work bandwidth will be seriously affected.

A microstrip part of SIW and microstrip line transformation form will have higher radiation loss.SIW waveguide section of this article is the relative dielectric constant is 2.65 FR4.its medium plate thickness is $0.5 \mathrm{~mm}$. Small to large is $5 \mathrm{~mm} \times 5.7$ $\mathrm{mm}$.In the medium plate have two rows of cylindrical hole, six in each row hole, a total of 12 hole. The distance between two rows of hole is $4.5 \mathrm{~mm}$, the diameter of the hole is $0.3 \mathrm{~mm}$, the distance between the hole and the hole is $0.4 \mathrm{~mm}$.

\section{B. Section Impedance Converter Theory and Design}

Because of the single section 1/4 impedance bandwidth for narrow-band converter work, so this paper uses the combination of multiple class impedance converter cascade to broadening the working frequency band.Insection impedance stepped impedance converter, if each impedance ladder produced by the reflected wave offset each other, its can make the matching band broadening ${ }^{[5]}$.

For an ideal section rheostat, after a variable resistance, on the transmission line $Z_{0}=1$, the reflection coefficient should be 0 .

Insert the transmission properties of two port with attenuation $\mathrm{L}$ said: 


$$
L=\frac{1}{\left|S_{12}\right|^{2}}=1+\frac{1-\left|S_{12}\right|^{2}}{\left|S_{12}\right|^{2}}=1+\xi
$$

Type(1) $\xi$ known as the excess attenuation,It represents and the difference between the state of ideal two-port network.As a matter of fact in a certain frequency range, excess attenuation $\xi$ can't all is 0 .As long as meet at the specified frequency band is less than a certain value.

The excess attenuation of section impedance converter:

$$
\xi=\frac{(R-1)^{2}}{4 R} \operatorname{COS}^{2 n} \theta
$$

Type (2) $\mathrm{R}$ for the variable resistance ratio, $\mathrm{n}$ for the rheostat session, $\theta$ for the transmission line segment length.

Analysis (1), (2) .In the center frequency $\omega=\omega_{0}, \theta=90^{\circ}$, $\cos \theta=0$.at this time $\xi=0$ Section for more ideal rheostat.When $\theta=\pi$, for the greatest excess attenuation. Which can determine the $\mathrm{Q}$ band, the size of section impedance transformer:

$L_{1}=3.67 \mathrm{~mm}, L_{2}=1.287 \mathrm{~mm}, L_{3}=2.1 \mathrm{~mm}, L_{4}=2.166 \mathrm{mma}=8.7 \mathrm{~m}$ $\mathrm{m}, a_{1}=5.7 \mathrm{~mm}, a_{2}=7.8 \mathrm{~mm}, a_{3}=7.7 \mathrm{~mm} b=2.85 \mathrm{~mm}, \quad b_{1}=2.7 \mathrm{~mm}$, $b_{2}=1.99 \mathrm{~mm}, b_{3}=1 \mathrm{~mm} b_{4}=0.5 \mathrm{~mm}$.

\section{TRANSITION DESIGN}

This article uses is SIW and conversion of the rectangular waveguide.Used to ensure that when the design within the required working frequency band characteristic impedance of the microstrip line and SIW equivalent impedance matching, reduce the reflection. Thus the bandwidth of the extension.

This structure as shown in figure 2.1, the waveguide model is made in two copper (orange) between designing a broad band of transition.The size of the copper is $72 \mathrm{~mm} * 32.4 \mathrm{~mm} * 28 \mathrm{~mm}$.Air rectangular(white) waveguide which is on the copper block dug a four step of waveguide.Figure 2.1, the green part is made of FR4 SIW waveguide. $\varepsilon_{r}=2.65$, the size is $5.7 \mathrm{~mm} * 5 \mathrm{~mm} * 0.5 \mathrm{~mm}$.Design of waveguide transition model is shown in figure 2.1.

Using Ansoft HFSS15.0 for the simulation analysis, main parameters and the optimization analysis, it is concluded that the optimized data for:

$L_{1}=3.67 \mathrm{~mm}, \quad L_{2}=1.287 \mathrm{~mm}, \quad L_{3}=2.1 \mathrm{~mm}, L_{4}=2.166 \mathrm{~mm}$, $a=8.7 \mathrm{~mm}, \quad a_{1}=5.7 \mathrm{~mm}, \quad a_{2}=7.8 \mathrm{~mm}, \quad a_{3}=7.7 \mathrm{~mm} b=2.85 \mathrm{~mm}$, $b_{1}=2.7 \mathrm{~mm}, \quad b_{2}=1.99 \mathrm{~mm}, \quad b_{3}=1 \mathrm{~mm} b_{4}=0.5 \mathrm{~mm}, \quad L_{\text {siw }}=10 \mathrm{~mm}$, $W_{\text {siw }}=4.5 \mathrm{~mm}, L_{\mathrm{h}}=1.5 \mathrm{~mm}, d=0.3 \mathrm{~mm}, s=0.4 \mathrm{~mm}$.

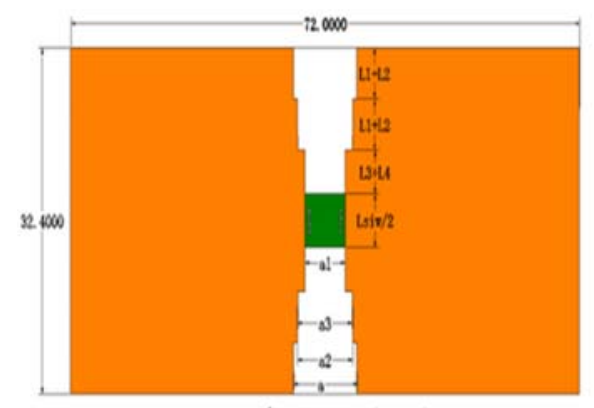

(a) Waveguide vertical view

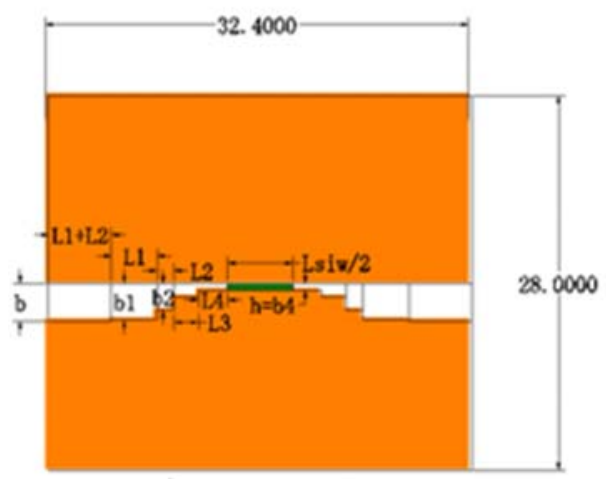

(b) Waveguide profile

FIGURE I. TRANSITION MODEL

Due to the effect of medium uniformity, actual length and the ideal optimization length will vary slightly, the transition of the final design is given priority to with the actual length optimization.

\section{Simulation Optimization And the RESult ANALYSIS}

Using Ansoft HFSS15.0 for the simulation analysis, The simulation results are shown in Figure 2, 3 and 4.

Figure 2 for the transition model with substrate integrated waveguide and without substrate integrated waveguide when the return loss of contrast.

For the presence of substrate integrated waveguide transition frequency almost does not have any effect, just an irregular impact on return loss. When without substrate integrated waveguide in the entire model, the return loss in most frequency are above $-10 \mathrm{~dB}$.when with substrate integrated in the entire model,Because the electromagnetic wave are limited to two rows of metal hole, this makes transverse magnetic wave does not exist.See in the figure 3 in the whole $30 \mathrm{GHZ}$ - $55 \mathrm{GHZ}$ range return loss are below - 10 $\mathrm{dB}$,So comprehensive consideration to insert substrate integrated waveguide can realize the design of wideband low-loss. 


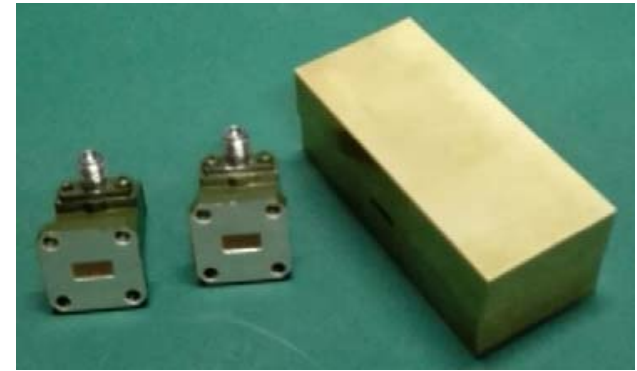

FIGURE V. TRANSITIONPHYSICAL

\section{The MeAsured Results And Analysis}

Physical transition by coaxial BJ400 conversion interface is connected to the vector network instrument for testing. The test results are shown in figure 6.

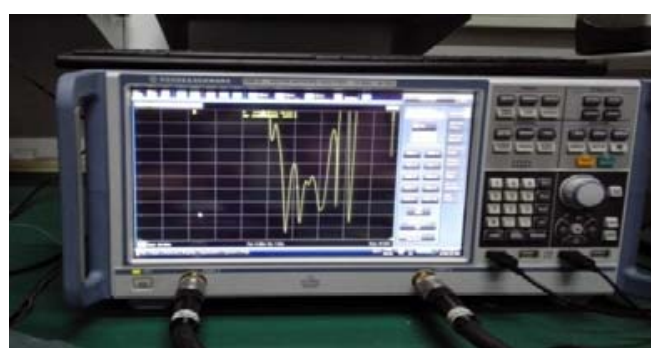

FIGURE VI. AGILENT TECHNOLOGIESN5230C 30GHZ-50GHZ

Figure 6 testing result compared with the results of simulation and comparison results as shown in figure 7 and figure 8 .

Figure 7 for transition simulation results with the measured results figure return loss.

Waveguide simulation return loss in 30-50 GHZ are below - $10 \mathrm{db}$,Compared with the simulation results. Waveguide spectrum test results in $30 \mathrm{GHZ}$ - $36 \mathrm{GHZ}, 53 \mathrm{GHZ}$ - $55 \mathrm{GHZ}$ return loss upward migration, in $36 \mathrm{GHZ}-53 \mathrm{GHZ}$ in the whole band return loss are below - $10 \mathrm{db}$.Due to the material and production process, error in the range of allowable error, the measured results and simulation results.

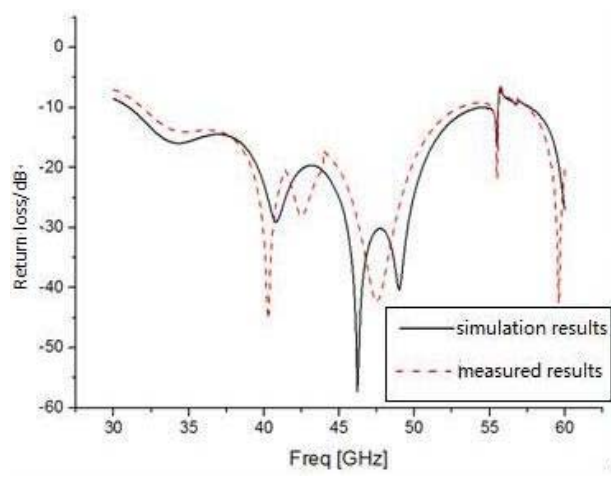

FIGURE VII. TRANSITION RETURN LOSS

Figure 8 for the transition simulation results compared with the measured results of insertion loss.

Waveguide physical is shown in figure 5. 

2014, 15(5): 108-116.

\section{[7] X. Chen and K. Wu. Substrate integrated waveguide filter: basic design
rules and fundamental structure features[J]. IEEE Microwave Magazine, \\ [7] X. Chen and K. Wu. Substrate integrated waveguide filter: basic design
rules and fundamental structure features[J]. IEEE Microwave Magazine,}

$55 \mathrm{GHZ}$ within $0.68 \mathrm{~dB}$,Compared with the simulation results. Waveguide spectrum test results in $30 \mathrm{GHZ}-40 \mathrm{GHZ}$ insertion loss downward shift.This is because the waveguide in the simulation itself as the ideal wall thickness, and in the actual production of waveguide is itself has certain thickness, is it going to affect insertion loss.in the simulation, the four step Angle as rectangular waveguide.in the actual process, because of the limitation of process precision made physical radian Angle,Cause certain influence to the insertion loss.

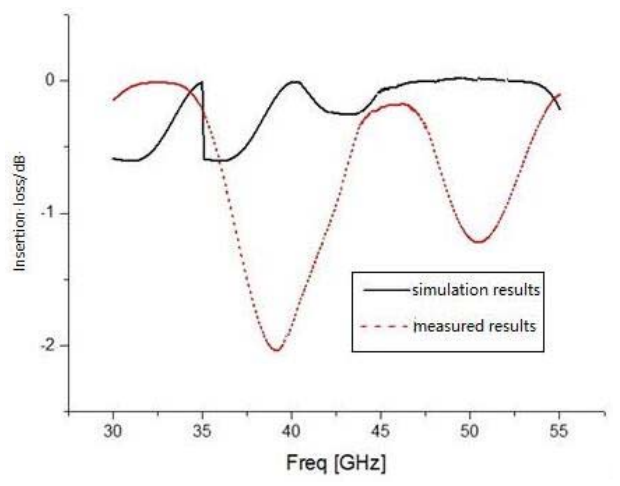

FIGURE VIII. TRANSITION INSERTION LOSS

\section{CONCLUSION}

Section impedance converter can be used to broaden the microwave circuit bandwidth realized the transition of the advantages of wide band design.At the same time in the middle to insert substrate integrated waveguide to realize transition of the low loss design.UsingAnsoft HFSS15.0 for the simulation analysis,Simulation and test of transition return loss under - 10 $\mathrm{db}$, basic covers $30 \mathrm{GHZ}$ - $55 \mathrm{GHZ}$ frequency band,Overall the transition and realize the microwave circuit design of wide band, low loss.

\section{ACKNOWLEDGMENTS}

(1) The Fund Project of Tarim University (TDZKQN201825)

(2) IoT Engineering Professional Comprehensive Reform Project of Tarim University (220101616)

\section{REFERENCE}

[1] Ahmet. Kayabasi. Analysis and synthesis of equilateral triangular ring microstrip sntenna using support vector machine[J]. Applied Computa-

[2] tional Electromagnetics Society Journal, 2018, 33(6): 616-624.

[3] N. Delmonte, L. Silvestril, M. Nozzi, L. Perregrinni. Compact half-

[4] mode SIW cavity filters designed by exploiting resonant mode control[C]. International Journal of RF and Microwave Computer-Aided Engineering, 2016, 26(1): 72-79.

[5] S. A. Pourghorban, K. Entesari. Ultra-miniature SIW cavity resonators and filters[J]. IEEE Transactions on Microwave Theory\&Techniques, 2015, 63(12): 4329-4340.

[6] R. Moro, S. Moscato, M. Bozzi, L. Perregrinni. Substrate integrated folded waveguide filter with out-of-band rejection controlled by resonant-mode suppression[J]. IEEE Microwave and Wireless Components Letters, 2015, 25(4): 214-216. 